\title{
Modulation of visual cortical excitability by working memory: effect of luminance contrast of mental imagery
}

\author{
Zaira Cattaneo ${ }^{1 *}$, Alberto Pisoni ${ }^{1}$, Costanza Papagno $^{1}$ and Juha Silvanto ${ }^{2}$ \\ Department of Psychology, University of Milano-Bicocca, Milano, Italy \\ Brain Research Unit, Low Temperature Laboratory and Advanced Magnetic Imaging Centre, Aalto University School of Science and Technology, Espoo, Finland
}

Edited by:

Gregor Thut, University of Glasgow, UK

Reviewed by:

Emily D. Grossman, University of

California Irvine, USA

Fang Fang, Peking University, China

*Correspondence:

Zaira Cattaneo, Department of

Psychology, University of Milano -

Bicocca, Piazza dell'Ateneo Nuovo 1,

20126 Milano, Italy.

email:zaira.cattaneo@unimib.it
Although much is known about the impact of stimulus properties such as luminance contrast, spatial frequency, and orientation on visually evoked neural activity, much less is known about how they modulate neural activity when they are properties of a mental image held in working memory (WM). Here we addressed this question by investigating how a parametric manipulation of an imagined stimulus attribute affects neuronal excitability in the early visual cortex. We manipulated luminance contrast, a stimulus property known to strongly affect the magnitude of neuronal responses in early visual areas. Luminance contrast modulated neuronal excitability, as assessed by the frequency of phosphenes induced by transcranial magnetic stimulation (TMS) with the exact nature of this modulation depending on TMS intensity. These results point to a strong overlap in the neuronal processes underlying visual perception and mental imagery: not only does WM maintenance selectively engage neurons which are tuned to the maintained attribute (as has previously been shown), but the extent to which those neurons are activated depends on the image contrast (as is the case with visually evoked responses). From a methodological viewpoint, these results suggest that assessment of visual cortical excitability using TMS is affected by the TMS intensity used to probe the neuronal population.

\section{INTRODUCTION}

The formation of representations in visuo-spatial working memory (VSWM) in the absence of bottom-up sensory input is referred to as visual mental imagery. The emerging view is that this process engages the same areas (and neurons) which are activated by visual presentation of that attribute (see Postle, 2006; D'Esposito, 2007 for discussion of sensory recruitment hypothesis; Chelazzi et al., 1993; Kosslyn et al., 1999; Sack et al., 2002, 2005; Harrison and Tong, 2009; Serences et al., 2009 for some evidence). Recent transcranial magnetic stimulation (TMS) studies have offered further support for this view. For example, phosphene induction is facilitated when the subject is engaging in visual imagery (Sparing et al., 2002), indicating that imagery increases visual cortical excitability, possibly through parietal modulation (Sack et al., 2008). Furthermore, phosphenes induced during mental imagery contain features of the memory item (Silvanto and Cattaneo, 2010), suggesting that mental imagery preferentially activates neurons which also play a causal role in visual perception of that feature.

Although much is known about the impact of various stimulus properties (such as luminance contrast and spatial frequency) on visually evoked neural activity, much less is known about how they modulate neural activity when they are properties of a mental image. This is an important question because previously reported facilitatory effects of mental imagery on phosphene perception could be due to factors such as attention (which has been shown to facilitate phosphene perception; Bestmann et al., 2007). In this view, the increased visual cortical excitability might reflect imageryinduced attentional facilitation of the maintained attributes rather than the actual content of the mental image. Here we addressed this question by investigating how a parametric manipulation of an imagined stimulus attribute affects the neuronal excitability of the early visual cortex. This was accomplished by manipulating luminance contrast, a stimulus property known to strongly affect the magnitude of neuronal responses in the early visual areas (e.g., Dean, 1981; Albrecht and Hamilton, 1982). For example, in human early visual areas the BOLD signal increases with stimulus contrast, and contrast discrimination thresholds correlate with the strength of V1 activation (e.g., Boynton et al., 1999; Buracas and Boynton, 2007).

Transcranial magnetic stimulation-induced phosphenes were used to assess neuronal excitability in the visual cortex (see, e.g., Boroojerdi et al., 2002; Sparing et al., 2002; Rauschecker et al., 2004; Silvanto et al., 2006; Bestmann et al., 2007; Romei et al., 2007, 2009; Marzi et al., 2009; for other studies using this phosphene approach). The logic was that, if the early visual cortex plays a role in the encoding of luminance information contained in mental images, then modulating the contrast of mental imagery should change visual cortical excitability and thus affect the likelihood of phosphene induction. Subjects were presented with a grating which they were asked to actively hold in VSWM; TMS was applied during the maintenance period. Subjects were asked to indicate whether they perceived the phosphene, and if so, to rate its intensity.

Four experiments were carried out. In Experiment 1, TMS was applied at phosphene threshold (PT). In order to ensure that subjects were actively engaged in active maintenance, catch trials assessing memory for stimulus contrast were included. In Experiments 2 and 3, TMS intensity and the presence/timing of the catch trials were manipulated. Specifically, we investigated whether the impact of imagery contrast on phosphene perception is dependent on the TMS intensity; this was accomplished 
by applying TMS at either PT or at a lower (90\% of PT) intensity. Furthermore, to investigate whether the catch trials affected the assessment of excitability in Experiment 1, no catch trials were included in Experiment 2, and in Experiment 3, the time point at which the catch trial is presented was changed. Additionally, a non-visual working memory (WM) control experiment (involving auditory memory) was carried out (Experiment 4) in order to determine any effects of non-visual cognitive load on phosphene perception.

\section{EXPERIMENT 1: THE IMPACT OF LUMINANCE CONTRAST OF MENTAL IMAGERY AND PASSIVE VIEWING ON PHOSPHENE PERCEPTION \\ METHOD \\ Participants}

Ten participants (five males, mean age $=23.5, \mathrm{SD}=3.2$ ) took part in Experiment 1. The experiment was undertaken with the understanding and written consent of each participant. The study was approved by the institutional review board of University of Milano-Bicocca, and all subjects were treated in accordance with the Declaration of Helsinki. All subjects were selected among a larger sample of volunteers with the inclusion criterion being the ability to consistently perceiving phosphenes with eyes open. All subjects were naïve to the aims of the study. The same criteria also hold for Experiments 2, 3, and 4 (see below).

\section{Visual stimuli and experimental task}

The stimuli were presented on a 17 -inch $(800 \times 600$ pixels $)$ monitor and consisted of a sinusoidal circular grating in a Gaussian envelop with a spatial frequency of 1 cycle/degree, and mean luminance of $6 \mathrm{~cd} / \mathrm{m}^{2}$. Its diameter was $7^{\circ}$ of visual angle and it was viewed from a distance of $57 \mathrm{~cm}$. Three different contrasts were used (Michelson contrasts of $0.1,0.5$, and 0.9 ). The orientation of the grating was $30^{\circ}$ from the vertical, either clockwise or counterclockwise. The center of the stimulus was at fixation.

"Visual Imagery" condition. Each trial began with a black fixation point appearing in the middle of the screen for $1000 \mathrm{~ms}$, followed by a blank screen for $500 \mathrm{~ms}$, after which the grating appeared for $500 \mathrm{~ms}$ (see Figure 1 for a timeline of each trial). In the "Visual Imagery" condition, this was followed by a 2-s maintenance period, during which a blank screen was presented. Subjects were asked to maintain a mental image of the grating, including its contrast. At the end of the maintenance period, a single pulse of TMS (at PT) was delivered over the early visual cortex. The TMS pulse was followed by a blank screen for $500 \mathrm{~ms}$, after which the sentence

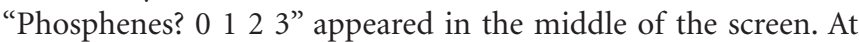
this point subjects were asked to verbally provide a rating of their phosphene perception using the following scale (adapted from the PAS-scale of Ramsøy and Overgaard, 2004): 0 = phosphene was absent; 1 = phosphene was weak; 2 = phosphene was almost clear; $3=$ phosphene was absolutely clear.

In order to ensure that subjects were maintaining the stimulus contrast in the Imagery condition, "catch" trials were included. On these trials, the maintenance period was followed by another grating that had either the same or different contrast as the memory item (the orientation was the same). The contrasts used in "Different trials" were different by $0.4-0.6$ in Michelson contrast, such that the contrast pairs were: 0.1 vs. $0.7 ; 0.5$ vs. $0.1 ; 0.9$ vs. $0.5 ; 0.1$ vs. 0.5 ; 0.5 vs. $0.9 ; 0.9$ vs. 0.3$)$. These contrast differences provided a catch trial performance in the region of $80 \%$, ensuring that the task was of sufficient difficulty. One Imagery block consisted of 48 trials, of

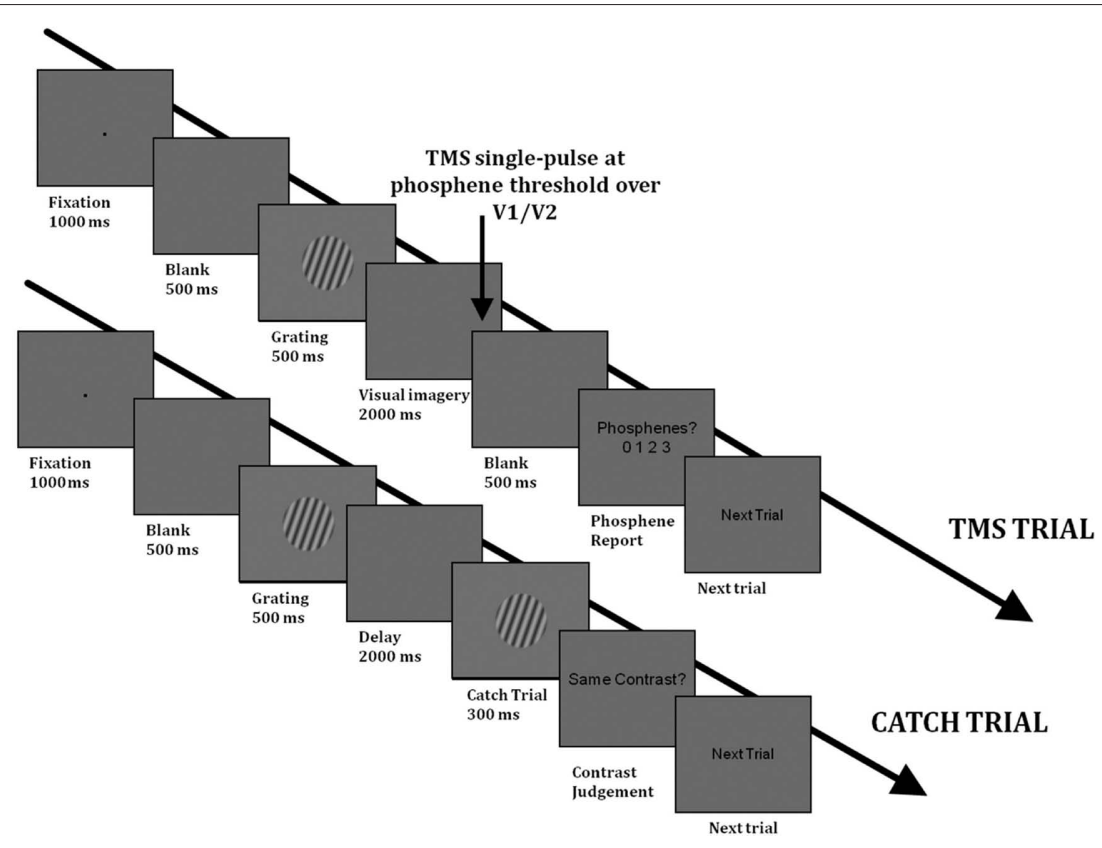

FIGURE 1 |Timelines of a TMS trial (above) and of a catch trial (below) in Experiment 1 in the visual imagery condition. The trials in the "Passive viewing" condition had the same structure as the TMS trials. In the "Passive viewing" condition, there were no catch trials and subjects were not asked to maintain a mental image of the grating. 
which 36 were TMS trials ( 12 for each contrast type) and 12 were catch trials ( 4 for each contrast type). The block was run four times; there were thus a total of 48 TMS trials per contrast.

"Passive" control condition. A "passive" control condition was also carried out in order to rule out the possibility that any impact of visual imagery on phosphene perception is not simply due to the presentation of the memory item. In this condition, the experimental trials were identical with the exception that subjects were not required to memorize the contrast of the grating during the maintenance period, and there were no catch trials. Each "Passive" block consisted of 36 trials, 12 for each contrast level. This block was also run four times.

The Imagery and Passive conditions were run consecutively. Half of the participants started with the Imagery condition, the other half with the Passive condition. Before the experiment subjects performed a series of practice trials (without TMS) to familiarize themselves with the task.

\section{TMS stimulation and site localization}

Transcranial magnetic stimulation was delivered by means of a Magstim Standard Rapid machine (Magstim, UK) via a 70-mm figure-of-eight-shaped coil. The early visual cortex was localized using a functional method in which the center of the coil is placed on the surface of the skull such that the stimulation elicits phosphenes that intrude the target location (for a discussion of this method, see Walsh and Pascual-Leone, 2003). In the present study, the phosphene location was such that it appeared in the center of the grating to be remembered. The starting point for the functional localization was the medial early occipital cortex, because stimulation of this site induces phosphenes intruding into both hemifields (as did the grating stimuli in the present study). For each subject, the PT was identified using a modified binary search algorithm (Tyrrell and Owens, 1988), an adaptive threshold finding algorithm, which gives a value for TMS intensity at which participants report phosphenes at 50\% of trials. In this method, the TMS intensity is increased or decreased according to the subject's report on the previous trial. The original upper boundary of the stimulation is $100 \%$ of stimulator output, and the lower limit is $0 \%$. TMS is applied at the value in the middle of the upper and lower boundary (i.e., if the lower and upper boundaries are 0 and $100 \%$, then TMS is applied at 50\%). If the participants reports seeing a phosphene, then the upper boundary is adjusted (such that the current TMS intensity becomes the upper boundary). If the participant does not see a phosphene, the lower boundary is adjusted, such that the current TMS intensity becomes the lower boundary. This is repeated until the two boundaries converge.

During the thresholding subjects had their eyes opened and viewed a computer screen with the same mean luminance as used in the main experiment. The mean TMS intensity required for inducing phosphenes was $63 \%$ of the maximum stimulator output $(\mathrm{SD}=19)$; during the experiment, subjects were stimulated at PT.

\section{RESULTS}

In two participants the rate of phosphene perception in both the passive viewing and the VSWM conditions was higher than 75\%, indicating that PT was underestimated and consequently the TMS intensity was too high. These subjects were removed from the analysis.
Figure 2 shows the proportion of "Phosphene present" judgments as a function of stimulus contrast $(0.1,0.5$, or 0.9$)$ and task condition (imagery or passive viewing). In this analysis, the three response types which indicate phosphene presence (i.e., "weak"; "almost clear," "absolutely clear") are combined. A $2 \times 3$ ANOVA, with task condition (imagery or passive viewing), and contrast level $(0.1,0.5,0.9)$ as within-subjects factors revealed a main effect of task condition $[F(1,7)=6.56, p=0.038]$ but no main effect of contrast $[F(2,14)=0.72, p=0.50]$ and no interaction $[F(2,41)=0.5$, $p=0.62]$. This analysis thus indicates that there was a general increase in "phosphene present" judgments associated with visual imagery which was not affected by stimulus contrast. Figure 3 shows the vividness ratings for the "Phosphene present" judgments for the Imagery and Passive viewing conditions. As can be seen in the figure, the vast majority of phosphenes were classified as "weak."

The performance on catch trials was on average $82 \%$ (low contrast: $82 \%$, middle contrast: $82 \%$, high contrast: $81 \%$ ). In a one-way ANOVA, catch trial accuracy was unaffected by the contrast of the memory item $[F(2,14)=0.04, p=0.96]$.

In summary, luminance contrast of mental imagery did not modulate the frequency of phosphene perception in Experiment 1.

\section{EXPERIMENT 2: THE IMPACT OF STIMULUS CONTRAST OF IMAGINED VISUAL STIMULI ON PHOSPHENE PERCEPTION IN THE ABSENCE OF CATCH TRIALS AND AT LOWER/HIGHER TMS INTENSITY}

In Experiment 1, a catch trial could be presented instead of a TMS pulse on any trial. This catch trial appeared at the same point in time as the TMS pulse on TMS trials. Therefore, in order to perform accurately on these catch trials, subjects had to monitor for the presence of catch trial on all trials. The impact of this may be that the knowledge of a possible catch trial stimulus may shift attention and neural activity away from visual imagery toward stimulus

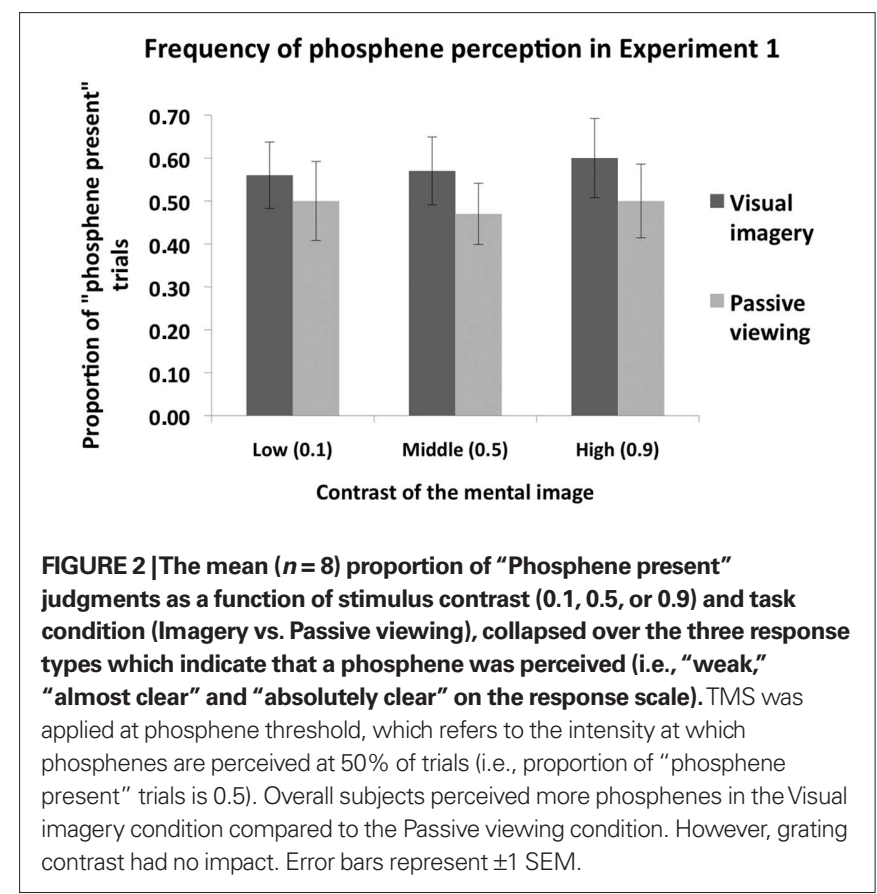


expectation, thus abolishing contrast dependency of visual imagery. In Experiment 2, this issue was investigated by removing catch trials from the experiment.

A second objective of Experiments 2 was to investigate whether stimulation intensity may have affected our results. There were two motivations for this. Firstly, an increase in phosphene perception by an experimental manipulation may be more likely to be found with lower TMS intensities, as there is more scope for phosphene perception to increase. A second motivation is that the interaction between TMS intensity and the neural activation state is not necessarily linear. TMS produces different neural and behavioral effects at low vs. high intensities (Reichenbach et al., 2011; Schwarzkopf et al., in press). It is thus important to investigate whether the lack of contrast modulation in Experiment 1 is due to suboptimal TMS intensity. We thus applied TMS, in a within-subjects design, at 100\% of PT (as in Experiment 1), and at 90\% of PT.

\section{PARTICIPANTS}

Eight participants (three males, mean age $=22.6$ years, $\mathrm{SD}=2.9$ ) took part in Experiment 2.

\section{VISUAL STIMULI AND EXPERIMENTAL TASK}

The visual stimuli and the task were the same as in Experiment 1, with the exception that catch trials were not included. Subjects were asked to maintain a mental image of the grating on each trial (as was

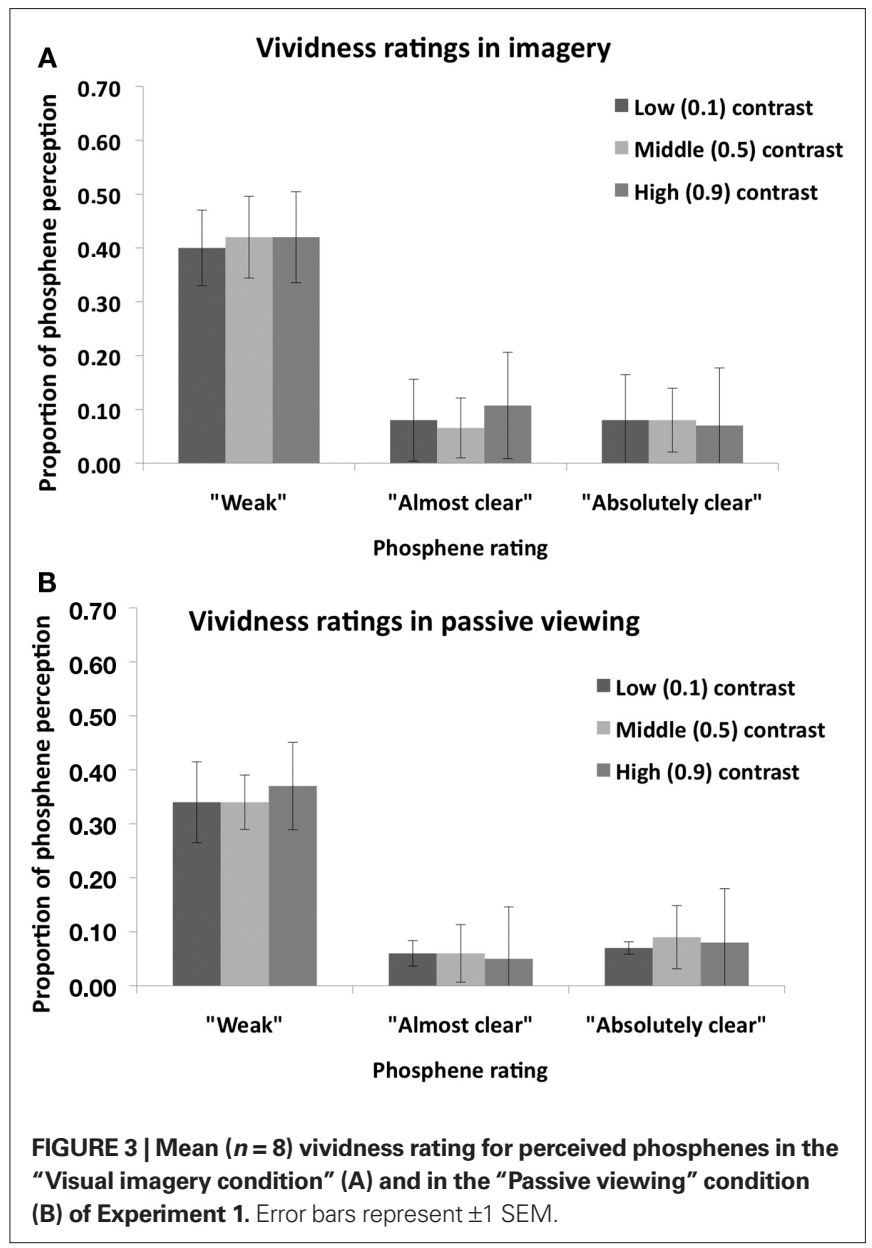

the case in Experiment 1). The experiment was run in four blocks of 48 trials (there were 32 trials per each stimulus contrast for each TMS intensity). The 100\% PT and 90\% PT TMS conditions were ran in separate bocks (two blocks for each TMS intensity).

\section{TRANSCRANIAL MAGNETIC STIMULATION}

Transcranial magnetic stimulation was carried out as in Experiment 1 . The mean PT was $62 \%(\mathrm{SD}=17)$ of the maximum stimulator output. In the experiment, TMS was applied either at 100\% of PT or at $90 \%$ of PT.

\section{RESULTS}

Figure 4 shows phosphene present judgments as a function of stimulus contrast and TMS intensity. Firstly, we analyzed the proportion of trials on which subjects perceived a phosphene as a function of contrast; in this analysis, the three response types (i.e., "weak"; "almost clear," "absolutely clear"), which indicate phosphene presence, are combined. A $2 \times 2$ ANOVA, with contrast level $(0.1,0.5,0.9)$ and TMS intensity as main factors $(100 \%$ of PT, $90 \%$ of PT), revealed a significant main effect of contrast $[F(1,7)=7.9, p=0.005]$, a significant main effect of TMS intensity $[F(1,7)=28.9, p=0.001]$ and a significant interaction between the two $[F(2,47)=4.9, p=0.02]$.

Post hoc (Sidak-adjusted) comparisons indicated that, when TMS was applied at $100 \%$ of PT, subjects perceived more phosphenes in the middle (0.5) contrast condition relative to the low $(0.1)$ contrast $(p<0.01)$ and high $(0.9)$ contrast $(p<0.01)$ conditions. There was no significant difference between the low and high contrast conditions $(p=0.89)$. Thus most phosphenes were induced in the middle contrast condition when TMS was applied

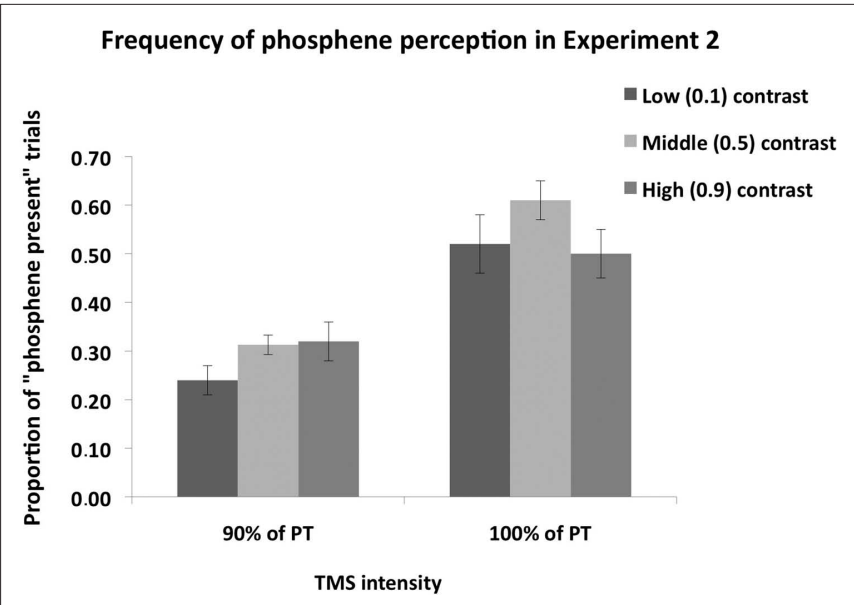

FIGURE 4 | Mean $(n=8)$ proportion of "Phosphene present" judgments in Experiment 2. The measure is collapsed over the three response types which indicate that a phosphene was perceived, i.e., "weak," "almost clear" and "absolutely clear" on the response scale rating. Error bars represent \pm 1 SEM. Mental imagery contrast interacted with TMS intensity. When TMS was applied at $90 \%$ of PT, significantly more phosphenes were perceived in the high and middle contrast conditions relative to the low contrast condition, indicating that an increase in luminance contrast enhances visual cortical excitability. When TMS was applied at $100 \%$ of PT, a significant difference was found between the low and middle contrast conditions, but not between the low and high contrast conditions. 
$100 \%$ of PT. A different pattern was found for the $90 \%$ of PT condition: relative to the low $(0.1)$ contrast condition, subjects perceived more phosphenes in the middle $(0.5)$ contrast $(p<0.05)$ and high $(0.9)$ contrast $(p<0.05)$ conditions. There was no significant difference between the middle and high contrast conditions $(p=0.82)$. Thus more phosphenes were induced in the middle and high contrast conditions relative to the low contrast condition. The vividness ratings for both TMS intensities are shown in Figure 5. As can be seen in the figure, the vast majority of phosphenes were classified as "weak."

In summary, mental imagery contrast modulated phosphene frequency, but the nature of this modulation depended on TMS intensity.

\section{EXPERIMENT 3: THE IMPACT OF STIMULUS CONTRAST OF IMAGINED VISUAL STIMULI ON PHOSPHENE PERCEPTION WITH DELAYED CATCH TRIALS AND AT LOWER/HIGHER TMS INTENSITY}

As discussed above, the timing of the catch trial presentation in Experiment 1 may have masked the impact of mental image contrast on visual cortical excitability. This is because accurate catch trial performance required the subjects to monitor for the presence of catch trial targets on all trials at the time when the phosphene was induced. In Experiment 3, this issue was investigated by

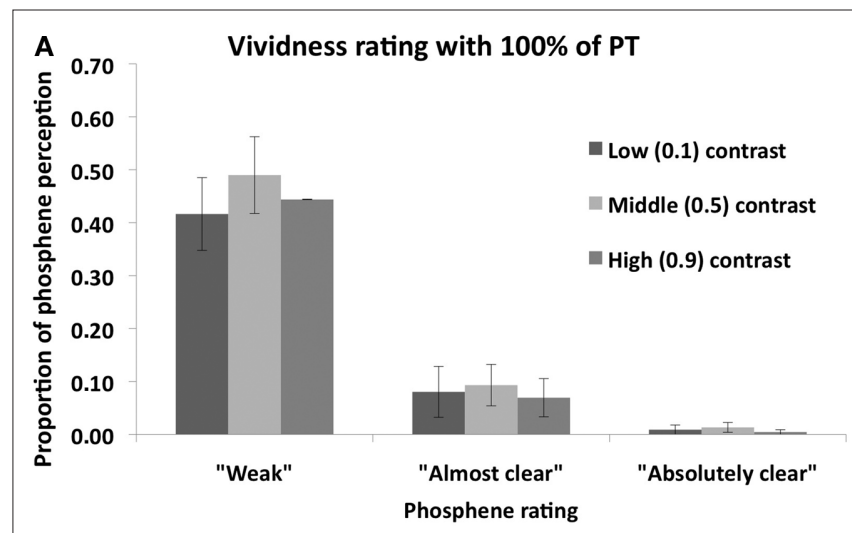

B

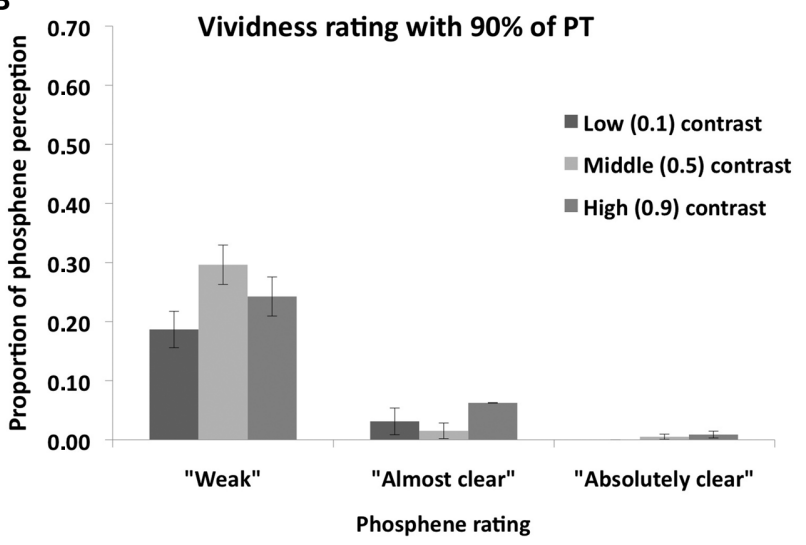

FIGURE 5 | Mean $(n=8)$ vividness ratings of perceived phosphenes in Experiment 2 for (A) 100\% PT condition; and (B) $90 \%$ PT condition. Error bars represent \pm 1 SEM. introducing a 1-s delay between the end of the maintenance period and the presentation of the catch trial target. This delay ensured that subjects were not required to monitor for the catch trial at the time when the phosphene was induced. As in Experiment 2, TMS was applied either at PT (i.e., 100\% of PT) or below PT (i.e., at $90 \%$ of PT).

\section{METHOD}

Participants

Seven participants (three males, mean age $=27$ years) took part in Experiment 3.

\section{Visual stimuli and experimental task}

The visual stimuli and the task were the same as in Experiment 1 , with the exception that only the low (0.1) and high (0.9) Michelson contrast targets were included. Subjects were asked to maintain a mental image of the grating on each trial. The experiment was run in four blocks of 40 trials; thus the whole experiment consisted of 160 trials. There were 32 trials for both stimulus contrasts at both TMS intensities (i.e., a total of 128 TMS trials). In addition there were 16 catch trials for both stimulus contrasts. The catch target could be of either same or different contrast as the memory item; if it was different, its contrast differed from the memory item by 0.4 (Michelson contrast). The $100 \%$ PT and 90\% PT TMS conditions were ran in separate bocks (consecutive two blocks for each TMS intensity). The order of these blocks was counterbalanced; one half of participants started with the 90\% PT condition and the other half started with the $100 \%$ PT condition. Subjects were asked to indicate whether they perceived a phosphene. In contrast to Experiments 1 and 2 , vividness ratings were not collected.

\section{Transcranial magnetic stimulation}

Transcranial magnetic stimulation was carried out as in Experiment 1 . The mean PT was $59 \%(\mathrm{SD}=21)$ of the maximum stimulator output. In the main experiment, TMS was applied either at $100 \%$ of PT or at $90 \%$ of PT.

\section{RESULTS}

Figure 6 shows phosphene present judgments as a function of stimulus contrast and TMS intensity. A $2 \times 2$ ANOVA, with contrast level $(0.1,0.9)$ and TMS intensity $(100 \%$ of PT, $90 \%$ of $\mathrm{PT})$ as main factors, revealed a significant main effect of contrast $[F(1,6)=10.29, p=0.02]$, a significant main effect of TMS intensity $[F(1,7)=25.5, p<0.001]$ and a significant interaction between the two $[F(2,27)=8.94, p=0.024]$. Post hoc (Sidak-adjusted) comparisons indicated that, when TMS was applied at $90 \%$ of PT, subjects perceived more phosphenes in the high (0.9) contrast condition than in the low $(0.1)$ contrast condition $(p<0.01)$; no such difference was found when TMS was applied at $100 \%$ of PT $(p=0.53)$.

Subject's mean performance on catch trials was: low contrast, 90\% PT TMS: 83\%; high contrast, 90\% PT TMS: 84\%; low contrast, 100\% PT TMS: 81\%; high contrast, 100\% PT TMS: 84\%.

In summary, mental imagery contrast modulated phosphene frequency when TMS was applied below PT (90\% of PT), but not when TMS was applied at $100 \%$ of PT. 


\section{EXPERIMENT 4: THE IMPACT OF AUDITORY SHORT-TERM MEMORY ON PHOSPHENE PERCEPTION}

It could be argued that any difference in phosphene frequency between the active imagery and passive viewing conditions in Experiment 1 is due to cognitive load being higher in the former. In this view, it is not the engagement of the visual system by mental imagery that affects phosphene perception, but rather the demand on cognitive resources by WM. To test this possibility, the impact of non-visual WM load on phosphene perception was investigated. In this study, involving auditory memory, subjects were asked to hold brief sounds in WM while phosphenes were induced.

\section{METHOD}

\section{Participants}

Eleven participants (five males, mean age $=24.1, \mathrm{SD}=2.1$ ) took part to Experiment 4. Ten of the subjects had taken part in Experiment 1.

\section{Auditory stimuli and experimental task}

Each trial began with a black fixation point appearing in the middle of the gray screen for $1000 \mathrm{~ms}$, followed by a blank screen for $500 \mathrm{~ms}$, after which a sound was delivered through headphones. The duration of the sound was either $400,500,600$, or $700 \mathrm{~ms}$. The sound was followed by a blank screen for $2000 \mathrm{~ms}$. In the "Auditory memory" condition, subjects were asked to memorize the sound duration. A single pulse of TMS at each subjects' individual PT was delivered over the early visual cortex. This was followed by a blank screen $(500 \mathrm{~ms})$, after which participants reported whether they had perceived a phosphene (as in Experiment 1). On catch trial, a test stimulus was presented instead of the TMS pulse; its duration could be either the same (in half of the trials) or different (differing for $100 \mathrm{~ms}$ ) than that of the memory item. Each block consisted of 32 trials, of which 24 were TMS trials and 8 were catch trials. The proportion of catch trials per block was the same as in Experiment 1. This block was run twice; there were thus a total of 48 TMS trials.

\section{Frequency of phosphene perception in Experiment 3}

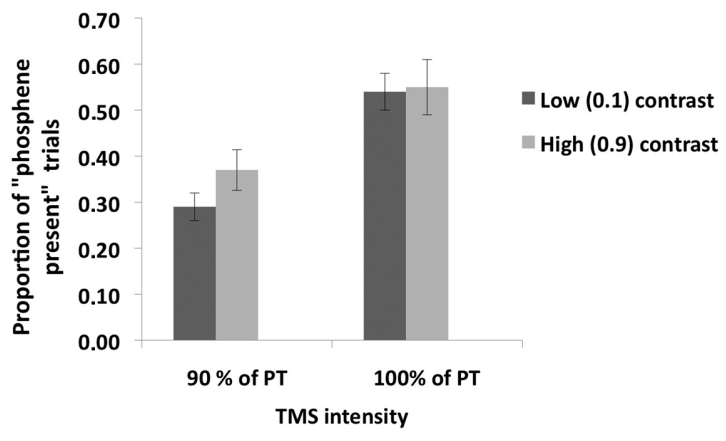

FIGURE 6 | Mean $(n=7)$ proportion of "Phosphene present" judgments in Experiment 3. As in Experiment 2, there was no difference in phosphene frequency between the low and high contrast stimuli when TMS was applied at $100 \%$ of phosphene threshold. When TMS was applied at $90 \%$ of PT, significantly more phosphenes were perceived during high contrast mental imagery than in the low contrast imagery condition, indicating that an increase in contrast enhanced visual cortical excitability. Error bars represent \pm 1 SEM.
"Passive" listening condition. In the passive listening condition, the trial structure was the same but subjects were not required to memorize the duration of the sound and there were no catch trials. This condition was run in one block of 48 trials.

Half of the participants started with the passive listening condition, the other half with the auditory memory condition.

\section{Transcranial magnetic stimulation}

Transcranial magnetic stimulation was carried out as in the experiments above. The mean PT was $57.8 \%$ of the maximum stimulator output $(\mathrm{SD}=11.5)$

\section{RESULTS}

Figure 7 shows the proportion of "Phosphene present" judgments in the Auditory memory and Passive listening conditions. In this analysis, the three response types indicating phosphene presence (i.e., responses 1, 2, and 3) are combined. A repeated-measures ANOVA with task condition (auditory memory vs. passive listening) as main factor found no significant difference between the two conditions, $F(1,10)=0.04, p=0.86$, suggesting that there was no general effect of memory load on phosphenes judgment. The mean catch trial performance was $75 \%$, a level similar to that in the VSWM condition (80\%).

Participants gave overall more " 1 " ratings (corresponding to weak phosphenes perception) compared to " 2 " and " 3 " ratings in both the auditory memory condition (rating " 1 " $=38.3 \%$; rating " 2 " $=11.9 \%$; rating " 3 " $=3.0 \%$ of the total TMS trials), and in the passive listening condition (rating " 1 " $=36.0 \%$; rating " 2 " $=10.8 \%$; rating " 3 " $=7.0 \%$ of the total TMS trials).

In summary, Experiment 4 showed that auditory WM did not modulate phosphene perception relative to passive auditory condition.

\section{DISCUSSION}

Our results show that the luminance contrast of mental imagery modulates the excitability of the visual cortex, and that the assessment of this modulation is affected by the TMS intensity with

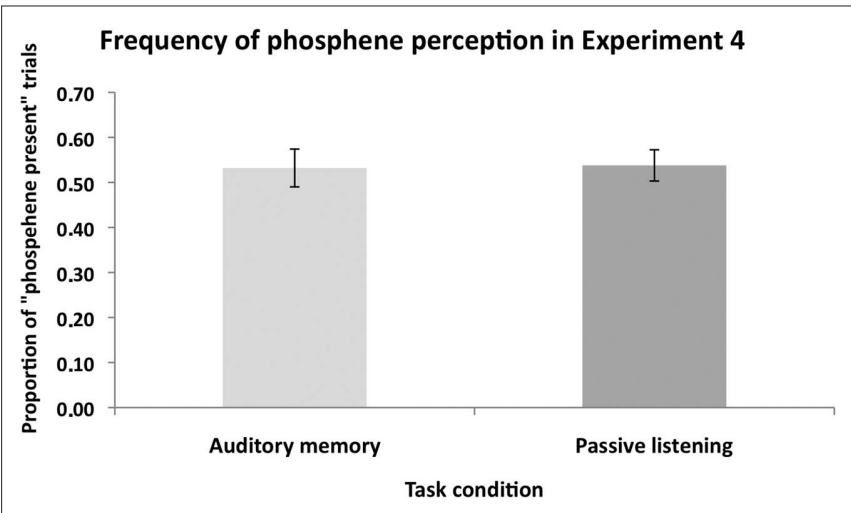

FIGURE 7 |The mean $(n=11)$ proportion of "Phosphene present" judgments in Experiment 4. There was no significant difference in the proportion of perceived phosphenes between the "auditory memory" and "passive listening" conditions. This shows that non-visual cognitive load did not modulate the excitability of the visual cortex. Error bars represent \pm 1 SEM 
which the neuronal populations are probed. In Experiment 1, phosphenes were perceived more frequently during visual mental imagery than in the "passive" viewing condition, indicating that visual imagery led to a general increase in visual cortical excitability. This is consistent with previous evidence (Sparing et al., 2002) and demonstrates that our VSWM paradigm engaged the early visual cortex. However, the frequency of phosphene perception was not modulated by the contrast of the mental image. This lack of modulation may have been due to the knowledge of a possible catch trial stimulus shifting attention and neural activity away from visual imagery toward stimulus expectation, thus abolishing contrast dependency of visual imagery. This may have occurred because the catch trial target was presented at the same time point at which the phosphene was induced on TMS trials. Thus the subjects were required to monitor for the presence of a catch trial target on all trials; the impact of divided attention may have masked the effect of mental imagery contrast on neuronal excitability. This possibility is supported by the findings that in Experiment 2 (in which catch trials were not included) and Experiment 3 (in which the catch trial targets were presented at a later time point such that no monitoring for catch trials was required), the frequency of phosphene perception was modulated by the mental image contrast. Finally, in Experiment 4, auditory imagery did not modulate phosphene perception, indicating that the increase in visual cortical excitability in Experiments 1-3 is not due to non-visual cognitive load.

\section{INTERACTION BETWEEN TMS INTENSITY AND IMAGERY CONTRAST: ASSESSMENT OF EXCITABILITY IS AFFECTED BY STIMULATION INTENSITY}

Interestingly, the impact of mental imagery contrast on phosphene perception depended on TMS intensity. In Experiment 2, when TMS was applied below PT, significantly more phosphenes were perceived in the middle and high contrast conditions relative to the low contrast conditions. This is roughly in line with a positive relationship between luminance contrast and excitability (although no increase from middle to high contrast was found; see next paragraph). In contrast, when TMS was applied at PT, a different pattern was observed. Significantly more phosphenes were perceived in the middle (0.5) contrast condition relative to the low (0.1) contrast condition as well as relative to the high (0.9) contrast condition. Furthermore, the significant difference between low and high contrast conditions, which was present when TMS was applied below PT, was not found.

The former pattern is perhaps more straightforward to explain. When contrast is increased from low to middle, neuronal excitability is increased and thus more phosphenes are perceived. The lack of any further excitability increase when moving from middle contrast to high contrast could be due to response saturation. In most V1 neurons the facilitation of neuronal firing resulting from a contrast increase is largest at relatively low contrasts. When stimulus contrast is already high, an additional increase has relatively small effect on neuronal responses. Neuroimaging evidence in human observers has shown that increasing the contrast from 0.5 to 0.9 Michelson contrast has a proportionally smaller impact on the activation level than increasing it from 0.1 to 0.4 (e.g., Boynton et al., 1996, 1999).
When TMS was applied at $100 \%$ of PT, the surprising finding is that phosphene perception was less frequent in the high contrast condition than in the middle contrast condition. It appears as if neural excitability increased from low to middle contrast condition, and then decreased from middle contrast to high contrast condition. However, a more parsimonious explanation of these results is that the assessment of neuronal excitability is affected by the strength of the external signal with which the neurons are probed. There is prior evidence on the non-linearity of TMS effects when stimulation intensity is manipulated. Moliadze et al. (2003) showed that, when applied concurrently with visually induced neural activity, low intensity TMS facilitated neural firing, whereas high intensity TMS had a suppressive effect. This difference between low vs. high intensity TMS effects may interact with the initial state of the stimulated neurons. For example, stimulation that can be described as "Low Intensity" at a baseline state of excitability might have a stronger impact when neurons are in a high state of excitation. In other words, the impact of "facilitatory low intensity" TMS may be more similar to that of "suppressive high intensity" TMS when excitability is increased. In the present study, if mental imagery contrast modulates neuronal excitability, then what can be described as "low intensity" and what as "high intensity" TMS would depend on stimulus contrast. Specifically, the transition from "facilitatory low intensity" stimulation to "suppressive high intensity" stimulation would occur at a lower TMS intensity for the high contrast stimulus than for lower contrasts (assuming a positive relationship between excitability and contrast). $100 \%$ of PT may be the transition point from facilitatory to suppressive effects for the high contrast mental stimulus; this may explain why the facilitation observed at $90 \%$ of PT relative to the low contrast stimulus is no longer found. For the middle contrast stimulus the transition would occur at a higher TMS intensity and thus the facilitation (relative to the low contrast stimulus) is still present at the higher intensity (i.e., 100\% of PT).

A similar interaction between TMS intensity and mental imagery contrast was also found in Experiment 3. In this experiment, a modulatory impact of mental imagery contrast on phosphene perception was found at the lower (90\% of PT) TMS intensity but not when TMS was applied at $100 \%$ of PT. This pattern of results replicates the findings of Experiment 2 (note that the "middle" contrast was not included in Experiment 3). It is worth noting that catch trials were included in Experiment 3 and thus one can be confident that subjects were indeed engaging in WM maintenance.

\section{CONTENT OF WM MAINTENANCE MODULATES VISUAL CORTICAL EXCITABILITY: CONTRAST RESPONSE FUNCTION OF MENTAL IMAGERY}

The finding that stimulus contrast had no effect on phosphene frequency when subjects passively viewed the visual stimuli ("Passive condition" in Experiment 1) demonstrates that results of Experiments 2 and 3 are due to VSWM maintenance rather than mere perception of the stimuli. That WM increases visual cortex neuronal excitability is consistent with the fMRI evidence (Soto et al., 2007) showing that the reappearance of a stimulus held in WM enhances activity in occipital areas known to encode the prior occurrence of stimuli. These findings are also consistent with prior TMS studies in which active maintenance of visual features has been shown to selectively increase the excitability of visual 
neurons engaged in VSWM (Silvanto and Cattaneo, 2010). When the impact of maintaining visual stimuli in WM has been assessed with behavioral paradigms rather than phosphenes, facilitations in the detection of the memory item have been found (Cattaneo et al., 2009; Schwarzkopf et al., 2010; Soto et al., 2010). Thus both phosphene studies and behavioral TMS studies indicate that neurons engaged in WM are more readily activated by TMS. In contrast, in conventional "passive" priming paradigms TMS has been found to impair the detection of the primed attribute (Cattaneo, 2010; Silvanto et al., 2010) and/or facilitate the detection of nonprimed attributes (Cattaneo et al., 2008, 2010). This is consistent with the evidence that mere stimulus repetition elicits a suppressive response in the same regions in which facilitation of neural activity is observed during WM maintenance (Soto et al., 2007).

A large number of studies have implicated visual cortical areas in visual imagery (see Postle, 2006 for review), and these regions have been shown to play a causal role in VSWM maintenance (Cattaneo et al., 2009). There is also more direct evidence indicating that the visual cortical activation changes induced by imagery can directly interact with information reaching awareness. As discussed above, maintaining the direction of a motion stimulus in VSWM affects the direction of moving phosphenes induced from the motionselective area V5/MT (Silvanto and Cattaneo, 2010). This indicates that the neurons which give rise to visual motion perception are also

\section{REFERENCES}

Albrecht, D. G., and Hamilton, D. B. (1982). Striate cortex of monkey and cat: contrast response function. $J$. Neurophysiol. 48, 217-237.

Bestmann, S., Ruff, C. C., Blakemore, C., Driver, J., and Thilo, K. V. (2007). Spatial attention changes excitability of human visual cortex to direct stimulation. Curr. Biol. 17, 134-139.

Boroojerdi, B., Meister, I. G., Foltys, H., Sparing, R., Cohen, L. G., and Töpper, R. (2002). Visual and motor cortex excitability: a transcranial magnetic stimulation study. Clin. Neurophysiol. 113, 1501-1504.

Boynton, G. M., Demb, J. B., Glover, G. H., and Heeger, D. J. (1999). Neuronal basis of contrast discrimination. Vision Res. 39, 257-269.

Boynton, G. M., Engel, S. A., Glover, G. H., and Heeger, D. J. (1996). Linear systems analysis of functional magnetic resonance imaging in human V1. J. Neurosci. 16, 4207-4221.

Buracas, G. T., and Boynton, G. M. (2007). The effect of spatial attention on contrast response functions in human visual cortex. J. Neurosci. 27, 93-97.

Cattaneo, L. (2010). Tuning of ventral premotor cortex neurons to distinct observed grasp types: a TMS-priming study. Exp. Brain Res. 207, 165-172.

Cattaneo, Z., Devlin, J. T., Salvini, F., Vecchi, T., and Silvanto, J. (2010). The causal role of category-specific neuronal representations in the left ventral premotor cortex $(\mathrm{PMv})$ in semantic processing. Neuroimage 49 , 2728-2734.

Cattaneo, Z., Rota, F., Vecchi, T., and Silvanto, J. (2008). Using statedependency of transcranial magnetic stimulation (TMS) to investigate letter selectivity in the left posterior parietal cortex: a comparison of TMS-priming and TMS-adaptation paradigms. Eur. J. Neurosci. 28, 1924-1929.

Cattaneo, Z., Vecchi, T., Pascual-Leone, A., and Silvanto, J. (2009). Contrasting early visual cortical activation states causally involved in visual imagery and short-term memory. Eur. J. Neurosci. 30, 1393-1400. Desimone, R. (1993). A neural basis for visual search inferior temporal cortex. Nature 363, 345-347.

Dean, A. F. (1981). The relationship between response amplitude and contrast for cat striate cortical neurons. J. Physiol. 318, 413-427.

D'Esposito, M. (2007). From cognitive to neural models of working memory. Philos. Trans. R. Soc. Lond., B, Biol. Sci. 362, 761-772.

Harrison, S. A., and Tong, F. (2009). Decoding reveals the contents of visual working memory in early visual areas. Nature 458, 632-635.

Kosslyn, S. M., Pascual-Leone, A., Felician, O., Camposano, S., Keenan, J. P., Thompson, W. L., Ganis, G., Sukel, K. E., and Alpert, N. M. (1999). The role of area 17 in visual imagery:
Chelazzi, L., Miller, E. K., Duncan, J., and

engaged in the WM maintenance (Silvanto and Cattaneo, 2010). The present results extend this literature by demonstrating that a parametric modulation of a stimulus attribute produces systematic changes in the excitability of the early visual cortex. In other words, not only does WM selectively engage neurons that are strongly activated by visual presentation of the maintained attribute, but also the extent to which those neurons are activated depends on strength of the mental image (in this case luminance contrast). This provides further evidence for the view that mental imagery is based on similar neuronal mechanisms as visual perception.

\section{CONCLUSION}

Our results show that luminance contrast of mental imagery modulates neuronal excitability in the early visual cortex. Not only does WM maintenance selectively engage neurons which are tuned to the maintained attribute (as has previously been shown), but the extent to which those neurons are activated depends on strength of the mental image (in this case luminance contrast). This suggests that the similarity in neuronal processes between visual perception and imagery extends to contrast response function and that the early visual cortex underlies the subjective experience of contrast in mental images. From a methodological viewpoint, these results suggest that assessment of visual cortical excitability using TMS is affected by the TMS intensity used to probe the neuronal population.

convergent evidence from PET and rTMS. Science 284, 167-170.

Marzi, C. A., Mancini, F., and Savazzi, S. (2009). Interhemispheric transfer of phosphenes generated by occipital versus parietal transcranial magnetic stimulation. Exp. Brain Res. 192 431-441.

Moliadze, V., Zhao, Y., Eysel, U., and Funke, K. (2003). Effect of transcranial magnetic stimulation on single-unit activity in the cat primary visual cortex. J. Physiol. 553(Pt 2), 665-679.

Postle, B. R. (2006). Working memory as an emergent property of the mind and brain. Neuroscience 139, 23-38.

Ramsøy, T.Z., and Overgaard, M. (2004). Introspection and subliminal perception. Phenomenol. Cogn. Sci. 3 , $1-23$.

Rauschecker, A. M., Bestmann, S., Walsh, V., and Thilo, K. V. (2004). Phosphene threshold as a function of contrast of external visual stimuli. Exp. Brain Res. 157, 124-127.

Reichenbach, A., Whittingstall, K., and Thielscher, A. (2011). Effects of transcranial magnetic stimulation on visual evoked potentials in a visual suppression task. Neuroimage 54 1375-1384.

Romei, V., Murray, M. M., Cappe, C., and Thut, G. (2009). Preperceptual and stimulus-selective enhancement of low-level human visual cortex excitability by sounds. Curr. Biol. 19, 1799-1805.
Romei, V., Murray, M. M., Merabet, L. B., and Thut, G. (2007). Occipital transcranial magnetic stimulation has opposing effects on visual and auditory stimulus detection: implications for multisensory interactions. $J$. Neurosci. 27, 11465-11472.

Sack, A. T., Camprodon, J. A., PascualLeone, A., and Goebel, R. (2005) The dynamics of interhemispheric compensatory processes in mental imagery. Science 308, 702-704.

Sack, A. T., Jacobs, C., De Martino, F. Staeren, N., Goebel, R., and Formisano, E. (2008). Dynamic premotor-toparietal interactions during spatial imagery. J. Neurosci. 28, 8417-8429.

Sack, A. T., Sperling, J. M., Prvulovic, D., Formisano, E., Goebel, R., Di Salle, F., Dierks, T., and Linden, D. E. (2002). Tracking the mind's image in the brain II: transcranial magnetic stimulation reveals parietal asymmetry in visuospatial imagery. Neuron 35 , 195-204.

Schwarzkopf, D. S., Silvanto, J., GilaieDotan, S., and Rees, G. (2010). Investigating object representations during change detection in human extrastriate cortex. Eur. J. Neurosci. 32, 1780-1787.

Schwarzkopf, D. S., Silvanto, J., and Rees, G. (in press). Stochastic resonance effects reveal the neural mechanisms of transcranial magnetic stimulation. J. Neurosci.

Serences, J. T., Ester, E. F., Vogel, E. K., and Awh, E. (2009).Stimulus-specific 
delay activity in human primary visual cortex. Psychol. Sci. 20, 207-214.

Silvanto, J., and Cattaneo, Z. (2010). Transcranial magnetic stimulation reveals the content of visual short-term memory in the visual cortex. Neuroimage 50, 1683-1689.

Silvanto, J., Lavie, N., and Walsh, V. (2006). Stimulation of the human frontal eye fields modulates sensitivity of extrastriate visual cortex. J. Neurophysiol.96, 941-945.

Silvanto, J., Schwarzkopf, D. S., GilaieDotan, S., and Rees, G. (2010). Differing causal roles for lateral occipital cortex and occipital face area in invariant shape recognition. Eur. J. Neurosci. 32, 165-171.
Soto, D., Humphreys, G. W., and Rotshtein, P. (2007). Dissociating the neural mechanisms of memorybased guidance of visual selection. Proc. Natl. Acad. Sci. U.S.A. 104, 17186-17191.

Soto, D., Wriglesworth, A., BahramiBalani, A., and Humphreys, G. W. (2010). Working memory enhances visual perception: evidence from signal detection analysis. J. Exp. Psychol. Learn. Mem. Cogn. 36, 441-456.

Sparing, R., Mottaghy, F. M., Ganis, G., Thompson, W. L., Topper, R., Kosslyn, S. M., and Pascual-Leone, A. (2002). Visual cortex excitability increases during visual mental imagery- a TMS study in healthy human subjects. Brain Res. 938, 92-97.

Tyrrell, R. A., and Owens, D. A. (1988). A rapid technique to assess the resting states of eyes and other threshold phenomena: the modified binary search (MOBS). Behav. Res. Methods Instrum. Comput. 20, 137-141.

Walsh, V., and Pascual-Leone, A. (2003). Transcranial Magnetic Stimulation: A Neurochronometrics of Mind. Cambridge, MA: MIT Press.

Conflict of Interest Statement: The authors declare that the research was conducted in the absence of any commercial or financial relationships that could be construed as a potential conflict of interest.
Received: 27 December 2010; accepted: 14 February 2011; published online: 25 February 2011.

Citation: Cattaneo Z, Pisoni A, Papagno $C$ and Silvanto J (2011) Modulation of visual cortical excitability by working memory: effect of luminance contrast of mental imagery. Front. Psychology 2:29. doi: 10.3389/fpsyg.2011.00029

This article was submitted to Frontiers in Perception Science, a specialty of Frontiers in Psychology.

Copyright (C) 2011 Cattaneo, Pisoni, Papagno and Silvanto. This is an open-access article subject to an exclusive license agreement between the authors and Frontiers MediaSA, which permits unrestricted use, distribution, and reproduction in any medium, provided the original authors and source are credited. 